\title{
A Two-Grid Alternate Strip-Based Domain Decomposition Strategy in Two-Dimensions
}

\author{
L. Angela Mihai ${ }^{1}$ and Alan W. Craig ${ }^{2}$ \\ 1 University of Durham, Department of Mathematical Sciences \\ (1.a.mihai@durham.ac.uk) \\ 2 University of Durham, Department of Mathematical Sciences \\ (alan.craig@durham.ac.uk)
}

Summary. The alternate strip-based iterative substructuring algorithms are preconditioning techniques for the discrete systems which arise from the finite element approximation of symmetric elliptic boundary value problems. The algorithms presented in this paper may be viewed as simple, direct extensions of the two disjoint subdomains case to the multiple domains decomposition with interior cross-points. The separate treatment of vertex points is avoided by dividing the original nonoverlapping subdomains into strip-subregions. Both scalability and efficiency are enhanced by alternating the direction of the strips.

\section{Introduction}

In domain decomposition (DD) work is concentrating both on the improvement of existing algorithms as well as on the development of new ones and it is mainly the treatment of the interface relations between subdomains that distinguishes one method from another (Chan and Mathew [1994], Smith et al. [1996], Xu and Zou [1998], Quarteroni and Valli [1999]). The goal of our work is to construct simple, efficient preconditioners with good parallelism and optimal convergence properties, which draw upon the strengths of both overlapping and non-overlapping DD methodologies. After the model problem is introduced, the rest of this paper is organised as follows: Section 2 is devoted to the description and analysis of the one level strip-based substructuring $\left(S B S_{2}\right)$ algorithm. Each strip is a union of non-overlapping subdomains and the global interface between subdomains is partitioned as a union of edges between strips and edges between subdomains which belong to the same strip. In Section 3 we derive and investigate the two-grid alternate stripbased substructuring $\left(A S B S_{2 g}\right)$ algorithms. The key ingredients are alternate strip-based solvers which generate algorithms in two stages and allow the use of efficient subdomain preconditioners such as a two-grid $V$ or $W$ cycle. We emphasise that a novel feature of our approach is that at each stage the direction of the strips changes and with it, the coupling between vertex points and 
edges. In Section 4 we illustrate the performance of the new two-grid strategy by some numerical estimates. The techniques introduced here (see also Mihai and Craig [2003]) extend in a straightforward manner to three-dimensional problems (Mihai and Craig [2004]). Once it is understood how and why it works, the alternate strip-based substructuring $(A S B S)$ strategy can be regarded more as a principle in DD and extended to more general problems defined on more complex geometries. For an extended discussion on this new strategy, in two and three dimensions, we also refer to Mihai [2004].

The Problem. We consider a second order, symmetric, coercive equation, with homogeneous Dirichlet boundary conditions, defined on a polygonal domain $\Omega \subset \mathbb{R}^{2}$. With the given domain we associate a uniform square grid $\Sigma^{h}$ of mesh-size $h$. The Galerkin finite element approximation generates the equivalent algebraic problem in the form of the linear system:

$$
A u=f,
$$

where the matrix $A$ is symmetric and positive definite and $f$ is the load vector. We are interested in the case when $A$ is ill-conditioned and a preconditioned conjugate gradient (PCG) method is employed for solving the original partial differential equation.

The DD Approach. Without loss of generality, we assume $\Omega$ to be of unit diameter. A DD without overlapping, of the domain $\Omega$, consists of a number of mutually disjoint open subdomains $\Omega_{i}$, such that: $\bar{\Omega}=\bigcup_{i=1}^{N} \bar{\Omega}_{i}$. Let all subdomains $\Omega_{i}$ be of size $H(h<H<1)$ in the sense that there exists constants $c$ and $C$ independent of $H$ and $h$ such that $\Omega_{i}$ contains a ball of diameter $c H$ and it is contained in a ball of diameter $C H$. Let also the coefficients of the original equation be either constants or piecewise constants. In the latter case the partition into disjoint subdomains is chosen in such a way that the jumps in the coefficients align with subdomain boundaries. We also assume that the triangulation $\Sigma^{h}$ is consistent with the original DD in the sense that each $\partial \Omega_{i}$ can be written as a union of boundaries of elements in $\Sigma^{h}$. Let $\Gamma$ denote the global interface between all subdomains $\left\{\Omega_{i}\right\}_{i=1}^{N}$. Then the linear system (1) can be written equivalently as:

$$
\left[\begin{array}{ll}
A_{I I} & A_{I E} \\
A_{I E}^{T} & A_{E E}
\end{array}\right]\left[\begin{array}{c}
u^{I} \\
u^{E}
\end{array}\right]=\left[\begin{array}{c}
f^{I} \\
f^{E}
\end{array}\right]
$$

where the indices $I$ and $E$ are associated with the nodes in $\Omega \backslash \Gamma$ and the nodes in $\Gamma$ respectively. By eliminating $u^{I}$, we obtain:

$$
S u^{E}=f_{S},
$$

where $S=A_{E E}-A_{I E}^{T} A_{I I}^{-1} A_{I E}$ is the Schur complement (SC) matrix and $f_{S}=f^{E}-A_{I E} A_{I I}^{-1} f^{I}$. The condition number $\kappa(S)$, although smaller than $\kappa(A)$, deteriorates with respect to the subdomain size $H$, the finite element 
mesh-size $h$, and the coefficients of the model problem (Le Tallec [1994]). Our work aims to solve the SC system (2) by constructing a parallel preconditioner $M$, via a new DD strategy augmented with two-grid iteration. The equation (1) gets solved by the following procedure:

(I) (Preprocessing) solve the equation with homogeneous Dirichlet boundary conditions on every subdomain $\Omega_{i} \subset \Omega$.

(II) $(P C G)$ solve the equation on the interface-boundary between all subdomains in $\Omega$.

(III) (Postprocessing) update the solution on every subdomain $\Omega_{i}$, using the boundary conditions given by $(I I)$.

Note that the preconditioned matrix $B^{-1} A$ has the same eigenvalues as the matrix $M^{-1} S$, plus the eigenvalue 1 .

\section{One-Level Strip-Based Substructuring}

We begin by associating the non-overlapping subdomains in the initial partition of $\Omega$, into strip-subregions $\Omega^{s}$, whose vertices are on the boundary $\partial \Omega$ and whose edges align with the edges of the original subdomains. Each strip is a union of non-overlapping subdomains and the global interface between subdomains is partitioned as a union of edges between strips (which include also all the cross points in the initial partition) and edges between subdomains which belong to the same strip (inside strips, the interface edges do not contain their end points), see Fig.1 left.

The One-Level Strip-Based Substructuring $\left(S B S_{2}\right)$ Algorithm. If $u^{k}$ is a given iteration, we define:

$$
u^{k+1} \leftarrow S B S_{2}\left(u^{k}, S, f_{S}\right)
$$

to be the new approximation for the solution to the SC problem when the following process is applied:

$$
u^{k+1} \leftarrow u^{k}+M^{-1}\left(f_{S}-S u^{k}\right),
$$

where $M$ is a preconditioner, such that the preconditioned system is symmetric and positive definite, hence it can also be used with CG acceleration. The new procedure for solving (2) can be described as follows:

$\left(\mathrm{II}_{1}\right)$ solve the one-dimensional equation with homogeneous Dirichlet boundary conditions on every edge between subdomains inside strips, with a preconditioner.

$\left(\mathrm{II}_{2}\right)$ solve the one-dimensional equation on every edge between strips, with a preconditioner.

$\left(\mathrm{II}_{3}\right)$ update the solution on every edge between subdomains inside strips, with Dirichlet boundary conditions from $\left(I I_{2}\right)$. 
For a survey of edge preconditioners, see e.g. Keyes and Gropp [1987]. We consider the elements (strip-edges, edges inside strips) of the boundary of a subdomain to be direct projections of the corresponding elements in $\Gamma$. Let:

$$
\Gamma=\bigcup_{k} \Gamma_{k} \cup \bigcup_{j} \Gamma^{j}
$$

where $\Gamma_{k}$ denotes a generic edge (an edge does not include its end points) inside strips, that is the interface between two adjacent subdomains inside a strip-subregion and $\Gamma^{j}$ denotes a generic strip-edge, that is the interface between two adjacent strip-subregions. We denote by $S_{h}^{0}\left(\Gamma_{k}\right)$ and $S_{h}^{0}\left(\Gamma^{j}\right)$ the subspace of the relevant boundary space consisting of functions whose support is contained in the corresponding edge. Let the following inner product:

$$
s\left(\mathbf{u}^{E}, \mathbf{v}^{E}\right)=\left(u^{E}\right)^{T} S v^{E}
$$

define the bilinear form associated with the Schur complement matrix under the standard nodal basis functions in $S_{h}(\Gamma)$. We decompose functions in $S_{h}^{0}(\Gamma)$ into $\mathbf{u}^{E}=\mathbf{u}^{e}+\mathbf{u}^{s}$, where

$$
\mathbf{u}^{e} \in V^{e}=\bigoplus_{k} S_{h}^{0}\left(\Gamma_{k}\right)
$$

and it is the solution of the following problem:

$$
s\left(\mathbf{u}^{e}, \mathbf{v}\right)=\left(\mathbf{f}_{S}, \mathbf{v}\right), \forall \mathbf{v} \in V^{e} .
$$

We solve for $\mathbf{u}_{k}^{e} \in S_{h}^{0}\left(\Gamma_{k}\right)$, on every edge $\Gamma_{k}$, the following local homogeneous Dirichlet problem:

$$
s\left(\mathbf{u}_{k}^{e}, \mathbf{v}\right)=\left(\mathbf{f}_{S}, \mathbf{v}\right), \forall \mathbf{v} \in S_{h}^{0}\left(\Gamma_{k}\right) .
$$

We denote by $\mathbf{u}^{s}=\mathbf{u}^{E}-\mathbf{u}^{e}$ the part of the solution $\mathbf{u}^{E}$ which lies in the orthogonal complement of $V^{e}$ in $S_{h}^{0}(\Gamma)$ :

$$
V^{s}=\left\{\mathbf{u} \in S_{h}^{0}(\Gamma): s(\mathbf{u}, \mathbf{v})=0, \forall \mathbf{v} \in V^{e}\right\} .
$$

Therefore $\mathbf{u}^{s}$ satisfies:

$$
s\left(\mathbf{u}^{s}, \mathbf{v}\right)=\left(\mathbf{f}_{S}, \mathbf{v}\right)-s\left(\mathbf{u}^{e}, \mathbf{v}\right), \forall \mathbf{v} \in S_{h}^{0}(\Gamma),
$$

or equivalently, by the definition of $V^{e}$,

$$
s\left(\mathbf{u}^{s}, \mathbf{v}^{s}\right)=\left(\mathbf{f}_{S}, \mathbf{v}\right)-s\left(\mathbf{u}^{e}, \mathbf{v}\right), \forall \mathbf{v} \in S_{h}^{0}(\Gamma) .
$$

Note that:

$$
s(\mathbf{u}, \mathbf{v})=s\left(\mathbf{u}^{e}, \mathbf{v}^{e}\right)+s\left(\mathbf{u}^{s}, \mathbf{v}^{s}\right)
$$

(here $\mathbf{v}^{e}$ and $\mathbf{v}^{s}$ are defined similarly as $\mathbf{u}^{e}$ and $\mathbf{u}^{s}$ respectively).

In the following lemma, every inequality can be proved by direct integration and with the help of the Cauchy inequality. 
Lemma 1. Let $\Omega \subset \mathbb{R}^{2}$ be the unit square and let $\Omega^{s}=(0,1) \times(0, H)$ be a strip-subregion of $\Omega$. For $\boldsymbol{u} \in H^{1}\left(\Omega^{s}\right)$, the following inequalities hold:

(i) if $\boldsymbol{u}$ is equal to zero along one short side of $\Omega^{s}$, then:

$$
\|\boldsymbol{u}\|_{L^{2}\left(\Omega^{s}\right)}^{2} \leq C|\boldsymbol{u}|_{H^{1}\left(\Omega^{s}\right)}^{2} .
$$

(ii) if $\boldsymbol{u}$ is equal to zero along one long side of $\Omega^{s}$, then:

$$
\|\boldsymbol{u}\|_{L^{2}\left(\Omega^{s}\right)}^{2} \leq C H^{2}|\boldsymbol{u}|_{H^{1}\left(\Omega^{s}\right)}^{2} .
$$

(iii) if $\Gamma^{j}$ is a long side of $\Omega^{s}$, then:

$$
\|\boldsymbol{u}\|_{L^{2}\left(\Gamma^{j}\right)}^{2} \leq C\left(\frac{1}{H}\|\boldsymbol{u}\|_{L^{2}\left(\Omega^{s}\right)}^{2}+H|\boldsymbol{u}|_{H^{1}\left(\Omega^{s}\right)}^{2}\right) .
$$

(iv) if $\boldsymbol{u} \in H^{1}(\Omega)$ then:

$$
\|\boldsymbol{u}\|_{L^{2}\left(\Omega^{s}\right)}^{2} \leq C H^{2}\left(\frac{1}{H}\|\boldsymbol{u}\|_{L^{2}(\Omega)}^{2}+|\boldsymbol{u}|_{H^{1}(\Omega)}^{2}\right) .
$$

Similar inequalities hold if we replace $\Omega^{s}$ by a square of side $H, \Omega_{i}^{s}=$ $(i H,(i+1) H) \times(0, H)$, and $\Omega$ by $\Omega^{s}$. $C$ denotes positive constants which are independent of the parameters $H$ and $h$. The actual value of these constants will not necessarily be the same in any two instances.

Theorem 1. For the $S B S_{2}$ algorithm with exact solvers on the subdomains, the condition number of the preconditioned system grows linearly as $1 / H$. For the case of discontinuous coefficients, the bounds are independent of the jumps in the coefficients as long as the jumps align with strip boundaries.

Corollary 1. For the case of homogeneous Dirichlet boundary conditions on $\partial \Omega$, if the domain $\Omega$ is reduced to at most two strip-subregions of width $H$, such that each strip is the reunion of $1 / H$ non-overlapping subdomains, then, for the $\mathrm{SBS}_{2}$ algorithm with exact solvers on the subdomains, the condition number of the preconditioned system is bounded independently of the partitioning parameters $H$ and $h$.

\section{Two-Grid Alternate Strip-Based Substructuring}

In this section we extend the $S B S_{2}$ algorithm, introduced in the previously, to a two-stage algorithm. In order to remove the factor $1 / H$ from the order of convergence, at each stage the direction of the strips changes and with it, the coupling between vertex points and edges (e.g. horizontal strips at the first stage, vertical strips at the second stage). Moreover, at the second stage, the calculations are carried out on a coarser grid. This can reduce considerably 
the amount of computational work needed to solve the problem to a particular accuracy. Let $\Sigma^{2^{p} h} \subset \cdots \subset \Sigma^{2 h} \subset \Sigma^{h}$ be a set of nested uniform square grids associated with the original domain $\Omega$, such that $1 \leq p \in \mathbb{N}$ and $2^{p} h \leq H$. To describe the two-grid algorithms, we introduce the following operators: the projection $P$ is an interpolation from grid $\Sigma^{2^{p} h}$ to grid $\Sigma^{h}$; the restriction from grid $\Sigma^{h}$ to grid $\Sigma^{2^{p} h}$ is defined as $R=P^{*}$. Finally, we shall also be using the notation: $S^{(1)}$ and $S^{(2)}$, for the coefficient matrix and $f_{S}^{(1)}$ and $f_{S}^{(2)}$, for the load vector of the linear system to be solved at the first and second stage respectively. Figure 1 shows the partition of the unit square $\Omega=(0,1) \times(0,1)$ into $1 / H$ disjoint, uniform strips $\Omega^{s}$, at two different stages.
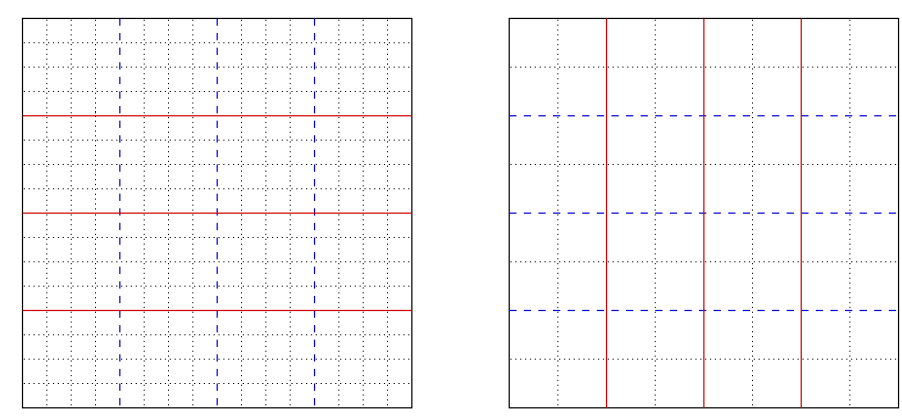

Fig. 1. The horizontal (left) and vertical (right) partition into strips of the domain $\Omega$, with two levels of mesh-refinement.

The Two-Grid Alternate Strip-Based Substructuring $\left(A S B S_{2 g}\right)$ Algorithm. Let $u^{k}$ be a given iteration, then $u^{k+1}$ is the result of the following V-cycle:

- $u^{k+\frac{1}{2}} \leftarrow S B S_{2}\left(u^{k}, S^{(1)}, f_{S}^{(1)}\right)$

- If $R^{(2)} \leftarrow f_{S}^{(2)}-S^{(2)} u^{k}, R_{c}^{(2)} \leftarrow R R^{(2)}$, its restriction to the coarse grid, then $w_{c}^{(2)} \leftarrow S B S_{2}\left(0, S_{c}^{(2)}, R_{c}^{(2)}\right)$, and its prolongation to the fine grid is $w^{(2)} \leftarrow P w_{c}^{(2)}$. We set

$$
u^{k+1} \leftarrow u^{k+\frac{1}{2}}+w^{(2)} .
$$

This procedure can be regarded as an additive Schwarz process between stages and expressed equivalently as:

$$
\begin{aligned}
u^{k+\frac{1}{2}} & \leftarrow u^{k}+M_{1}^{-1}\left(f_{S}^{(1)}-S^{(1)} u^{k}\right) \\
w^{(2)} & \leftarrow M_{2}^{-1}\left(f_{S}^{(2)}-S^{(2)} u^{k}\right) \\
u^{k+1} & \leftarrow u^{k+\frac{1}{2}}+w^{(2)},
\end{aligned}
$$

where $M_{1}$ and $M_{2}$ are preconditioners. The preconditioned system, which is symmetric and can be used with CG acceleration, can be written as: 


$$
M_{2 g}^{-1} S=M_{1}^{-1} S+M_{2}^{-1} S
$$

Note that at the coarse-grid level, if the mesh-size is equal to $H$, then only equations corresponding to the interface between strip-subregions need to be solved. Therefore, instead of defining the coarse-grid solver on the whole global interface $\Gamma$ between subdomains, we can consider only one-dimensional coarsesolvers defined on the edges between strip-subregions, then alternate the strips at the fine stage. We note that for problems in three dimensions, the possibility of reducing the size of the coarse solver from three to only two dimensions seems to offer an advantage (in forthcoming Mihai and Craig [2004]).

The performance of the $A S B S_{2 g}$ method is illustrated by the following result. Its proof is based on the observation that the preconditioner can be interpreted as a two-level overlapping Schwarz method, where every overlapping subdomain is the union of two adjacent subdomains that share the same edge.

Theorem 2. For the $A S B S_{2 g}$ method, if exact solvers are used for the subproblems, the condition number of the preconditioned system is bounded independently of the partitioning parameters $H$ and $h$.

\section{Numerical Estimates}

Example 1. Consider the model problem

$$
\begin{aligned}
& -\nabla \cdot \alpha(x) \nabla \mathbf{u}(x)=\mathbf{f}(x), \text { in } \Omega=(0,1) \times(0,1) \\
& \mathbf{u}(x)=0, \text { on } \partial \Omega,
\end{aligned}
$$

discretized by piecewise linear finite elements. In the computations, at each stage the unit square $\Omega$ is partitioned into $N=1 / H^{2}$ equal squares; $\alpha(\cdot)$ is 1 (for the Poisson equation) or random constants inside each subdomain. For the interface edges, the coefficients are the average values of all the subdomains adjacent to that interface. The mesh-parameter is $h$ for the fine grid and $H$ for the coarse-grid. The iteration counts are calculated for $10^{-4}$ reduction in error. All computations were carried out in Matlab.

Discussion. In Table 1, for the $S B S_{2}$ algorithm, the condition number of the preconditioned SC system grows like $1 / H$ and remains bounded independently of the mesh-size $h$. In Table 2, for $A S B S_{2 g}$, the condition number of the preconditioned SC system is less than 2 . The bounds are also independent of the jumps in the coefficients as long as the jumps align with subdomain boundaries. 
Table 1. Condition number and iteration counts for the $S B S_{2}$ algorithm.

\begin{tabular}{r|rr|rr|rr|rr}
\hline $\mathrm{N} \mid$ & $1 / \mathrm{h}=32$ & 64 & 128 & $\mid$ & 256 \\
\hline 4 & 1.3403 & 4 & 1.3408 & 4 & 1.3387 & 4 & 1.3376 & 4 \\
\hline 16 & 1.8739 & 6 & 1.8717 & 6 & 1.8755 & 6 & 1.8755 & 6 \\
\hline 64 & 3.2879 & 8 & 3.2801 & 8 & 3.2826 & 8 & 3.2916 & 8 \\
\hline 256 & 6.3364 & 12 & 6.3376 & 12 & 6.3276 & 12 & 6.3208 & 12 \\
\hline
\end{tabular}

Table 2. Condition number and iteration counts for the additive $A S B S_{2 g}$ algorithm.

\begin{tabular}{|c|c|c|}
\hline $\mathrm{N} \mid 1 / \mathrm{h}=32$ & $64 \mid 128$ & 256 \\
\hline \multicolumn{3}{|c|}{\begin{tabular}{l|l|l|l|l|l|l|l|}
4 & 1.2565 & 4 & 1.2582 & 4
\end{tabular}} \\
\hline \multicolumn{3}{|c|}{\begin{tabular}{|l|l|l|l|l|l|l|l|}
1.3467 & 4 & 1.3362 & 4 & 1.3284 & 1.32654
\end{tabular}} \\
\hline \multicolumn{3}{|c|}{ 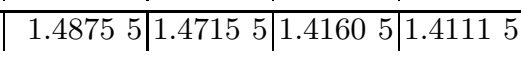 } \\
\hline \multicolumn{3}{|c|}{ 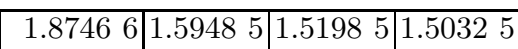 } \\
\hline
\end{tabular}

\section{References}

T. F. Chan and T. P. Mathew. Domain decomposition algorithms. In Acta Numerica 1994, pages 61-143. Cambridge University Press, 1994.

D. E. Keyes and W. D. Gropp. A comparison of domain decomposition techniques for elliptic partial differential equations and their parallel implementation. SIAM J. Sci. Stat. Comput., 8(2):s166-s202, 1987.

P. Le Tallec. Domain decomposition methods in computational mechanics. In J. T. Oden, editor, Computational Mechanics Advances, volume 1 (2), pages 121-220. North-Holland, 1994.

L. Mihai. A Class of Alternate Strip-Based Domain Decomposition Methods for Elliptic PDEs. PhD thesis, in preparation. University of Durham, U.K., 2004.

L. Mihai and A. Craig. Alternate strip-based substructuring algorithms for elliptic PDEs in two-dimensions. submitted, 2003.

L. Mihai and A. Craig. Alternate strip-based substructuring algorithms for elliptic PDEs in three-dimensions. submitted, 2004.

A. Quarteroni and A. Valli. Domain Decomposition Methods for Partial Differential Equations. Oxford Science Publications, 1999.

B. F. Smith, P. E. Bjørstad, and W. Gropp. Domain Decomposition: Parallel Multilevel Methods for Elliptic Partial Differential Equations. Cambridge University Press, 1996.

J. Xu and J. Zou. Some nonoverlapping domain decomposition methods. SIAM Review, 40:857-914, 1998. 